Trinity University

Digital Commons @ Trinity

Physics and Astronomy Faculty Research

Physics and Astronomy Department

8-2010

\title{
Green's Functions and the Adiabatic Hyperspherical Method
}

Seth T. Rittenhouse

Nirav P. Mehta

Trinity University, nmehta@trinity.edu

Chris H. Greene

Follow this and additional works at: https://digitalcommons.trinity.edu/physics_faculty

Part of the Physics Commons

\section{Repository Citation}

Rittenhouse, S.T., Mehta, N.P., \& Greene, C.H. (2010). Green's functions and the adiabatic hyperspherical method. Physical Review A - Atomic, Molecular, and Optical Physics, 82(2), 022706. doi: 10.1103/ PhysRevA.82.022706

This Article is brought to you for free and open access by the Physics and Astronomy Department at Digital Commons @ Trinity. It has been accepted for inclusion in Physics and Astronomy Faculty Research by an authorized administrator of Digital Commons @ Trinity. For more information, please contact jcostanz@trinity.edu. 


\title{
Green's functions and the adiabatic hyperspherical method
}

\author{
Seth T. Rittenhouse, ${ }^{1,2}$ N. P. Mehta, ${ }^{1,3}$ and Chris H. Greene ${ }^{1}$ \\ ${ }^{1}$ Department of Physics and Joint Institute for Laboratory Astrophysics (JILA), University of Colorado, Boulder, Colorado 80309, USA \\ ${ }^{2}$ Institute for Theoretical Atomic, and Molecular Physics (ITAMP), Harvard-Smithsonian Center for Astrophysics, \\ Cambridge, Massachusetts 02138, USA \\ ${ }^{3}$ Department of Physics, Grinnell College, Grinnell, Iowa 50112, USA
}

(Received 14 May 2010; published 12 August 2010)

\begin{abstract}
We address the few-body problem using the adiabatic hyperspherical representation. A general form for the hyperangular Green's function in $d$ dimensions is derived. The resulting Lippmann-Schwinger equation is solved for the case of three particles with $s$-wave zero-range interactions. Identical particle symmetry is incorporated in a general and intuitive way. Complete semianalytic expressions for the nonadiabatic channel couplings are derived. Finally, a model to describe the atom loss due to three-body recombination for a three-component Fermi gas of ${ }^{6} \mathrm{Li}$ atoms is presented.
\end{abstract}

DOI: 10.1103/PhysRevA.82.022706

PACS number(s): 34.50.-s, 31.15.xj, 67.85.Lm

\section{INTRODUCTION}

In recent years, there has been extensive theoretical and experimental interest in the area of few-body physics, most notably in the famous effect first predicted in 1970 by Efimov [1]. Efimov studied a three-boson system with short-range two-body interactions in which each two-body system is infinitesimally close to forming a bound state; that is, the $s$-wave scattering length is infinite, or at least very large in magnitude. Quantitatively, Efimov and later others found that this effect is described by a simple wave function in the hyperspherical representation [2-5]. A quantitative understanding of three-body scattering [4,6] has given experiments the tools to examine three-body processes in dilute gas systems and has led to a wealth of experimental evidence for the Efimov effect [7-12]. More recently, predictions relating to the four-body loss rate [13] have given another means of characterizing an Efimov resonance. The experimental realization of these predictions swiftly followed $[12,14]$.

The sticking point of the adiabatic hyperspherical method lies in solving the adiabatic Schrödinger equation. Often solving this $(d-1)$-dimensional equation is as hard as solving the total $d$-dimensional Schrödinger equation in the first place. Having a variety of methods available is therefore helpful. The benefit of using the adiabatic hyperspherical method comes from the simple final interpretation that can often be applied to the resulting coupled set of one-dimensional equations in the hyperradius [15]. For instance, in the three-body problem, if two particles can form a bound state, then one of the resulting scattering channels consists of an atom and a dimer colliding. In the adiabatic hyperspherical method, this type of fragmentation channel arises naturally as one of the discrete solutions to the hyperangular equations. In this paper, we derive the hyperangular Green's function for an arbitrary $d$-dimensional system, which can then be used in a hyperangular Lippmann-Schwinger equation to extract the adiabatic hyperradial potential curves.

This article is arranged as follows. In Sec. II we derive a general form of the hyperangular Green's function for a $d$ dimensional system. In Sec. III the Green's function is applied to the problem of three particles with regularized, zero-range, $s$-wave interactions. Section IV applies this result to the three lowest hyperfine states of ${ }^{6} \mathrm{Li}$ and gives a simple description of the scaling behavior of three-body recombination events that result in trap losses. Finally, in Sec. V we summarize the results and suggest further avenues of inquiry.

\section{THE HYPERANGULAR GREEN'S FUNCTION}

The adiabatic hyperspherical method has proven useful for analyzing many few-body systems [16-21]. The heart of this method lies in treating the overall size of the system, the hyperradius $R$ defined by $\sqrt{\mu} R=\sqrt{\sum_{i=1}^{d} \mu_{i} x_{i}^{2}}$, as an adiabatic parameter. Here $\mu_{i}$ is the mass scale associated with the $i$ th Cartesian coordinate and $\mu$ is the reduced mass associated with the hyperradius. For a system of $N$ particles, $x_{i}$ denote the $d=3 N-3$ Cartesian components needed to specify the relative positions of the $N$ particles. In this representation, the total wave function is written as

$$
\Psi(R, \Omega)=\sum_{n} R^{-(d-1) / 2} F_{n}(R) \Phi_{n}(R ; \Omega),
$$

where the adiabatic eigenfunctions $\Phi_{n}(R ; \Omega)$ satisfy the fixed$R$ Schrödinger equation

$$
\left[\frac{\hbar^{2}}{2 \mu} \frac{\Lambda^{2}}{R^{2}}+V(R, \Omega)\right] \Phi_{n}(R ; \Omega)=u_{n}(R) \Phi_{n}(R ; \Omega),
$$

Here, $\Lambda$ is the grand angular momentum operator defined by

$$
\begin{gathered}
\Lambda^{2}=-\sum_{i<j} \Lambda_{i j}^{2}, \\
\Lambda_{i j}=x_{i} \frac{\partial}{\partial x_{j}}-x_{j} \frac{\partial}{\partial x_{i}} .
\end{gathered}
$$

Inserting Eq. (1) into the full (time-independent) Schrödinger equation takes a $d$-dimensional partial differential equation to a set of coupled one-dimensional differential equations:

$$
\begin{aligned}
& {\left[-\frac{\hbar^{2}}{2 \mu}\left(\frac{d^{2}}{d R^{2}}-\frac{(d-3)(d-1)}{4 R^{2}}\right)+u_{n}(R)\right] F_{n}(R)} \\
& -\frac{\hbar^{2}}{2 \mu} \sum_{m}\left[2 P_{n m} \frac{d}{d R}+Q_{n m}\right] F_{m}(R)=E F_{n}(R) .
\end{aligned}
$$


The nonadiabatic coupling matrices $P$ and $Q$ in Eq. (2) are defined as

$$
\begin{aligned}
P_{m n} & =\left\langle\Phi_{m}(R ; \Omega) \mid \frac{\partial}{\partial R} \Phi_{n}(R ; \Omega)\right\rangle, \\
Q_{m n} & =\left\langle\Phi_{m}(R ; \Omega) \mid \frac{\partial^{2}}{\partial R^{2}} \Phi_{n}(R ; \Omega)\right\rangle .
\end{aligned}
$$

The integrals in Eqs. (6) and (7) are taken only over the $d-1$ hyperangles collectively denoted $\Omega$.

Approximate solutions can be found by solving the uncoupled system of equations, which are referred to as the adiabatic approximation:

$$
\begin{aligned}
& {\left[-\frac{\hbar^{2}}{2 \mu} \frac{d^{2}}{d R^{2}}+\frac{\hbar^{2}}{2 \mu} \frac{(d-3)(d-1)}{4 R^{2}}\right.} \\
& \left.\quad-\frac{\hbar^{2}}{2 \mu} Q_{n n}+u_{n}(R)\right] F_{n}(R)=E F_{n}(R)
\end{aligned}
$$

The ground-state eigenenergy Eq. (8) is a variational upper bound to the exact ground-state energy from Eq. (5). Another variant of this method is frequently denoted the Born-Oppenheimer approximation, with the diagonal correction $-\frac{\hbar^{2}}{2 \mu} Q_{n n}(R)$ to the potential $u_{n}(R)$ omitted. These two approximations will be the main focus of this paper, while the nonadiabatic couplings $P_{n m}(R)$ will be used to describe Landau-Zener-Stueckelberg transitions between the different hyperradial channels $u_{n}(R)$. Once the adiabatic potentials have been found, much of the intuition of simple onedimensional Schrödinger quantum mechanics can be brought to bear upon the problem. Unfortunately, obtaining these potentials can be prohibitively difficult in many problems; the development of efficient ways to calculate them is desirable.

This section derives the free space hyperangular Green's function for an arbitrary $d$-dimensional space such as an $N$-body system with $d=3(N-1)$ with the center-of-mass coordinate removed. This Green's function can then be used to recast Eq. (2) into an integral Lippmann-Schwinger (LS) equation. The $d$-dimensional Laplacian written in hyperspherical coordinates is given in Ref. [18] as

$$
\nabla^{2}=\frac{1}{R^{(d-1) / 2}} \frac{\partial^{2}}{\partial R^{2}} R^{(d-1) / 2}-\frac{(d-1)(d-3)}{4 R^{2}}-\frac{\Lambda^{2}}{R^{2}} .
$$

The hyperangular Green's function is given as the solution to

$$
\left[\Lambda^{2}-v(v+d-2)\right] G^{v}\left(\Omega, \Omega^{\prime}\right)=\delta^{d}\left(\Omega-\Omega^{\prime}\right) .
$$

Here $\Omega$ stands for the $d-1$ hyperangular coordinates needed to describe the surface of a $d$-dimensional hypersphere, and $\delta^{d}\left(\Omega-\Omega^{\prime}\right)$ is the Dirac $\delta$ function in the hyperangular coordinates, i.e., $\delta\left(\Omega-\Omega^{\prime}\right)=0$ if $\Omega \neq \Omega^{\prime}$ and $\int \delta^{d}\left(\Omega-\Omega^{\prime}\right)$ $d \Omega=1$. The Green's function can be found in several forms, including the full hyperspherical harmonic expansion [22], and has been given in closed form by Szmytkowski [23].

The simplest derivation of the Green's function relies on the completeness of hyperspherical harmonics:

$$
\sum_{\lambda \mu} Y_{\lambda \mu}^{*}\left(\Omega^{\prime}\right) Y_{\lambda \mu}(\Omega)=\delta^{d}\left(\Omega-\Omega^{\prime}\right)
$$

The function $Y_{\lambda \mu}$ is the solution to the eigenvalue equation

$$
\Lambda^{2} Y_{\lambda \mu}(\Omega)=\lambda(\lambda+d-2) Y_{\lambda \mu}(\Omega) .
$$

Here $\lambda$ is the hyperangular momentum quantum number, and $\mu$ enumerates the degenerate states. These functions are generally expressed as products of Jacobi polynomials for any number of dimensions and are thoroughly described by a number of authors (see Refs. [18,24] for some examples). They are simply an extension of normal spherical harmonics to a higher dimension.

Equation (11) can be used in conjunction with Eq. (12) to find $G^{v}\left(\Omega, \Omega^{\prime}\right)[22]$ :

$$
G^{\nu}\left(\Omega, \Omega^{\prime}\right)=\sum_{\lambda \mu} \frac{Y_{\lambda \mu}^{*}\left(\Omega^{\prime}\right) Y_{\lambda \mu}(\Omega)}{\lambda(\lambda+d-2)-v(\nu+d-2)} .
$$

Unfortunately, eigenfunction expansions of Green's function often have slow convergence with respect to the number of eigenfunctions, making them unsuitable for numerical calculations. The closed form of the Green's function from Ref. [23] is given as

$$
G^{\nu}\left(\Omega, \Omega^{\prime}\right)=\frac{-\pi}{(d-2) S_{d} \sin \pi \nu} C_{\nu}^{(d-2) / 2}\left(-\hat{R} \cdot \hat{R}^{\prime}\right),
$$

where $C_{v}^{\alpha}$ is a Gegenbauer function, $S_{d}$ is the surface area of the $d$-dimensional unit hypersphere $S_{d}=\int d \Omega=$ $2 \pi^{d / 2} / \Gamma(d / 2)$, and $\hat{R} \cdot \hat{R}^{\prime}$ is the cosine of the angle between the two normalized hypervectors $\hat{R}$ and $\hat{R}^{\prime}$. Here $v$ is defined by Eq. (10). While Eq. (14) has a pleasing, compact form, it is often divergent at critical points. For instance if $v$ is noninteger valued, then $G^{v}\left(\Omega, \Omega^{\prime}\right)$ diverges as $\hat{R} \cdot \hat{R}^{\prime} \rightarrow 1$.

For these reasons, it is convenient to find a third form of the Green's function. The first step in this derivation relies on the division of the total $d$-dimensional space into two subspaces. For the purposes of this work, we will assume that the dimension of the two subspaces are both greater than 2 , i.e., $d_{1}, d_{2} \geqslant 2$. The two subspaces are each described by subhyperspherical coordinates. The two resulting subhyperradii can then be related to the total hyperradius as

$$
\begin{aligned}
& R_{1}=R \sin \alpha, \\
& R_{2}=R \cos \alpha, \\
& 0 \leqslant \alpha \leqslant \pi / 2 .
\end{aligned}
$$

Reference [24] details how the hyperangular momentum can be written in terms of the subhyperangular momenta as in Eq. (17). With the following definitions:

$$
\begin{aligned}
& \Lambda_{1}^{2} Y_{\lambda_{1} \mu_{1}}\left(\Omega_{1}\right)=\lambda_{1}\left(\lambda_{1}+d_{1}-2\right) Y_{\lambda_{1} \mu_{1}}\left(\Omega_{1}\right), \\
& \Lambda_{2}^{2} Y_{\lambda_{2} \mu_{2}}\left(\Omega_{2}\right)=\lambda_{2}\left(\lambda_{2}+d_{2}-2\right) Y_{\lambda_{2} \mu_{2}}\left(\Omega_{2}\right),
\end{aligned}
$$

the Green's function can be expanded using the completeness of the subhyperspherical harmonics [viz., Eq. (18)]. Substituting the expansion in Eq. (18) into Eq. (10), we find that the latter is satisfied if and only if Eq. (19) is satisfied; $\delta\left(\alpha-\alpha^{\prime}\right)$ is a Dirac $\delta$ function and the denominator on the left-hand side of Eq. (19) arises from the hyperangular volume element 
associated with the angle $\alpha$ (see Refs. [18,23] for details).

$$
\begin{aligned}
& \boldsymbol{\Lambda}^{2}=\frac{-1}{(\sin \alpha)^{\left(d_{1}-1\right) / 2}(\cos \alpha)^{\left(d_{2}-1\right) / 2}} \\
& \times \frac{\partial^{2}}{\partial \alpha^{2}}(\sin \alpha)^{\left(d_{1}-1\right) / 2}(\cos \alpha)^{\left(d_{2}-1\right) / 2} \\
& +\frac{\boldsymbol{\Lambda}_{1}^{2}+\left(d_{1}-1\right)\left(d_{1}-3\right) / 4}{\sin ^{2} \alpha}+\frac{\boldsymbol{\Lambda}_{2}^{2}+\left(d_{2}-1\right)\left(d_{2}-3\right) / 4}{\cos ^{2} \alpha} \\
& -\frac{(d-1)(d-3)+1}{4} \text {. } \\
& G\left(\Omega, \Omega^{\prime}\right)=\sum_{\lambda_{1} \mu_{1}} \sum_{\lambda_{2} \mu_{2}} g\left(\alpha, \alpha^{\prime}\right) Y_{\lambda_{1} \mu_{1}}^{*}\left(\Omega_{1}^{\prime}\right) Y_{\lambda_{1} \mu_{1}}\left(\Omega_{1}\right) \\
& \times Y_{\lambda_{2} \mu_{2}}^{*}\left(\Omega_{2}^{\prime}\right) Y_{\lambda_{2} \mu_{2}}\left(\Omega_{2}\right), \\
& \frac{\delta\left(\alpha-\alpha^{\prime}\right)}{(\sin \alpha)^{d_{1}-1}(\cos \alpha)^{d_{2}-1}} \\
& =\left[\frac{-1}{(\sin \alpha)^{d_{1}-1}(\cos \alpha)^{d_{2}-1}} \frac{\partial}{\partial \alpha}(\sin \alpha)^{d_{1}-1}(\cos \alpha)^{d_{2}-1} \frac{\partial}{\partial \alpha}\right. \\
& +\frac{\lambda_{1}\left(\lambda_{1}+d_{1}-2\right)}{\sin ^{2} \alpha}+\frac{\lambda_{2}\left(\lambda_{2}+d_{2}-2\right)}{\cos ^{2} \alpha} \\
& -v(v+d-2)] g_{\lambda_{1}, \lambda_{2}}^{d_{1}, d_{2}}\left(v ; \alpha, \alpha^{\prime}\right) \text {. }
\end{aligned}
$$

The general one-dimensional Green's function for any differential equation of the Sturm-Liouville form Eq. (19) is

$$
g_{\lambda_{1}, \lambda_{2}}^{d_{1}, d_{2}}\left(v ; \alpha, \alpha^{\prime}\right)=\frac{-f_{\lambda_{1} \lambda_{2} v}^{+}\left(\alpha_{<}\right) f_{\lambda_{1} \lambda_{2} v}^{-}\left(\alpha_{>}\right)}{(\sin \alpha)^{d_{1}-1}(\cos \alpha)^{d_{2}-1} W\left[f^{+}, f^{-}\right]},
$$

where $W\left[f^{+}, f^{-}\right]=f^{+} f^{-\prime}-f^{-} f^{+\prime}$ is the Wronskian [25] and $\alpha_{<(>)}=\min \left(\alpha, \alpha^{\prime}\right)\left[\max \left(\alpha, \alpha^{\prime}\right)\right]$. The functions $f^{+}(\alpha)$ and $f^{-}(\alpha)$ are regular at $\alpha=0$ and $\alpha=\pi / 2$, respectively, and satisfy the homogeneous version of Eq. (19). The solutions $f^{+}(\alpha)$ and $f^{-}(\alpha)$ are given in Ref. [26] as

$$
\begin{aligned}
f_{\lambda_{1} \lambda_{2} v}^{( \pm)}(\alpha)= & \left(\sin ^{\lambda_{1}} \alpha \cos ^{\lambda_{2}} \alpha\right) \\
& \times{ }_{2} F_{1}\left(\frac{\lambda_{1}+\lambda_{2}-v}{2}, \frac{v+\lambda_{1}+\lambda_{2}+d-2}{2} ; \frac{2 \lambda_{ \pm}+d_{ \pm}}{2} ; \frac{1 \mp \cos 2 \alpha}{2}\right),
\end{aligned}
$$

$$
\begin{aligned}
& W\left[f_{\lambda_{1} \lambda_{2} v}^{+}, f_{\lambda_{1} \lambda_{2} v}^{-}\right] \\
& \quad=\frac{-2 \Gamma\left(\frac{2 \lambda_{1}+d_{1}}{2}\right) \Gamma\left(\frac{2 \lambda_{2}+d_{2}}{2}\right)}{(\sin \alpha)^{d_{1}-1}(\cos \alpha)^{d_{2}-1} \Gamma\left(\frac{v+\lambda_{1}+\lambda_{2}+d-2}{2}\right) \Gamma\left(\frac{\lambda_{1}+\lambda_{2}-v}{2}\right)},
\end{aligned}
$$

where ${ }_{2} F_{1}(a, b ; c, x)$ is a hypergeometric function, $\lambda_{+}=\lambda_{1}$, $d_{+}=d_{1}, \lambda_{-}=\lambda_{2}$, and $d_{-}=d_{2}$.

\section{THE THREE-BODY PROBLEM WITH ZERO-RANGE INTERACTIONS}

In this section we show the utility of the Green's function developed in the previous section by applying it to the threebody problem with regularized, zero-range, $s$-wave, pseudopotential interactions. This problem has been well studied by a variety of researchers [4,27-29]. The full Hamiltonian for the untrapped system is given by

$$
H_{\mathrm{tot}}=\sum_{i=1}^{3}-\frac{\hbar^{2}}{2 m_{i}} \nabla_{i}^{2}+\sum_{i>j} V_{i j}\left(r_{i j}\right),
$$

where $\vec{r}_{i}$ is the position of the $i$ th particle, and $\nabla_{i}^{2}$ is the Laplacian for $\vec{r}_{i}$. The interaction is given by

$$
V_{i j}\left(r_{i j}\right)=\frac{4 \pi \hbar^{2} a_{i j}}{2 \mu_{i j}} \delta^{(3)}\left(\vec{r}_{i j}\right) \frac{\partial}{\partial r_{i j}} r_{i j},
$$

where $a_{i j}$ is the $s$-wave scattering length between particles $i$ and $j$, and $\mu_{i j}$ is the two-body reduced mass, $\mu_{i j}=$ $m_{i} m_{j} /\left(m_{i}+m_{j}\right)$. The pseudopotential defined in this way applies the Bethe-Peierls boundary condition to the two-body wave function as $r \rightarrow 0$, i.e., $\psi(r) \rightarrow\left(1-a_{i j} / r\right) C$ for some constant $C$ [30]. The center of mass can be removed from this system by converting to a system of Jacobi vectors. Jacobi vectors are created for this system by considering the separation vector between two of the three particles and then a second vector from the center of mass of that two-body system to the third. The final vector is then just the center-of-mass coordinate. The choice of Jacobi vectors is not unique. Here we will need to consider three different Jacobi coordinate parametrizations, each of which is convenient for describing one of the three possible two-body interactions $V\left(r_{i j}\right)$. In the "odd-man-out" notation these are given by

$$
\begin{gathered}
\vec{\rho}_{1}^{(k)}=\left(\vec{r}_{i}-\vec{r}_{j}\right) / d_{k}, \\
\vec{\rho}_{2}^{(k)}=d_{k}\left(\frac{m_{i} \vec{r}_{i}+m_{j} \vec{r}_{j}}{m_{i}+m_{j}}-\vec{r}_{k}\right), \\
\vec{r}_{\mathrm{c} . \mathrm{m} .}=\frac{\left(m_{1} \vec{r}_{1}+m_{2} \vec{r}_{2}+m_{3} \vec{r}_{3}\right)}{m_{1}+m_{2}+m_{3}}, \\
d_{k}^{2}=\frac{\left(m_{k} / \mu\right)\left(m_{i}+m_{j}\right)}{m_{1}+m_{2}+m_{3}},
\end{gathered}
$$

where $\mu$ is the three-body reduced mass

$$
\mu=\sqrt{\frac{m_{1} m_{2} m_{3}}{m_{1}+m_{2}+m_{3}}} .
$$

The total Hamiltonian can be rewritten in terms of the Jacobi coordinates and the center of mass as

$$
\begin{gathered}
H_{\mathrm{tot}}=H+H_{\mathrm{c} . \mathrm{m} .}, \\
H_{\text {c.m. }}=\frac{-\hbar^{2}}{2 M} \nabla_{\mathrm{c} . \mathrm{m} .}^{2}, \\
H=-\frac{\hbar^{2}}{2 \mu} \sum_{i=1}^{2} \nabla_{\rho_{i}}^{2}+\sum_{i>j} V_{i j}\left(r_{i j}\right) .
\end{gathered}
$$

Transforming the Jacobi coordinate piece of the Hamiltonian in Eq. (27) into hyperspherical coordinates using Eqs. (9) and (25) yields

$$
\begin{aligned}
H= & -\frac{\hbar^{2}}{2 \mu} \frac{1}{R^{5 / 2}} \frac{\partial^{2}}{\partial R^{2}} R^{5 / 2}+\frac{15 \hbar^{2}}{8 \mu R^{2}}+\frac{\hbar^{2} \Lambda^{2}}{2 \mu R^{2}} \\
& +\sum_{i<j} V_{i j}\left(d_{k} \rho_{1}^{(k)}\right) .
\end{aligned}
$$


To apply the adiabatic hyperspherical formulation, the hyperangular adiabatic Schrödinger equation must be solved:

$$
\left[\Lambda^{2}+\frac{2 \mu R^{2}}{\hbar^{2}} \sum_{i<j} V_{i j}\left(d_{k} \rho_{1}^{(k)}\right)-v(v+4)\right] \Phi(R ; \Omega)=0 .
$$

This can now be accomplished with the use of the hyperangular Green's function, Eq. (18), in the Lippmann-Schwinger (LS) equation,

$$
\begin{aligned}
\Phi(R ; \Omega)= & -\frac{2 \mu R^{2}}{\hbar^{2}} \int d \Omega^{\prime} G^{v}\left(\Omega, \Omega^{\prime}\right) \\
& \times\left[\sum_{i<j} V_{i j}\left(d_{k} \rho_{1}^{(k) \prime}\right)\right] \Phi\left(R ; \Omega^{\prime}\right),
\end{aligned}
$$

where $\vec{\rho}_{1}^{(k) \prime}$ is the $k$ th Jacobi vector parametrized by $\left\{R, \Omega^{\prime}\right\}$. Because the system has been constrained to have a constant hyperradius, this is effectively a bound-state problem; note that Eq. (30) has been assumed here to have no noninteracting solution at the chosen value of $v$. The hyperradial Hamiltonian from Eq. (5) in the absence of the nonadiabatic couplings $P$ and $Q$ is given in terms of the hyperangular eigenvalue $v$ as

$$
\begin{gathered}
H_{R}=\frac{-\hbar^{2}}{2 \mu} \frac{\partial^{2}}{\partial R^{2}}+U_{n}(R), \\
U_{n}(R)=\frac{\hbar^{2}}{2 \mu}\left[\frac{\left(v_{n}+2\right)^{2}-1 / 4}{R^{2}}-Q_{n n}(R)\right] .
\end{gathered}
$$

To evaluate the integrals in the LS equation, the Green's function from Eq. (18) is expressed in terms of the appropriate Jacobi coordinate set for each interaction term in the sum, with the hyperangles defined as

$$
\Omega^{(k)}=\left\{\omega_{1}^{(k)}, \omega_{2}^{(k)}, \alpha^{(k)}\right\},
$$

where $\omega_{i}^{(k)}$ represents the spherical polar angular coordinates for $\vec{\rho}_{i}^{(k)}$. The remaining hyperangle $\alpha^{(k)}$ is defined as in Eq. (15), i.e.,

$$
\begin{aligned}
& \rho_{1}^{(k)}=R \sin \alpha^{(k)}, \\
& \rho_{2}^{(k)}=R \cos \alpha^{(k)} .
\end{aligned}
$$

With this choice of hyperangles, it is clear that $d_{1}=d_{2}=3$ and the hyperspherical sub-harmonics $Y_{\lambda_{i} \mu_{i}}^{(i)}\left(\Omega_{i}\right)$ in Eq. (18) reduce to normal spherical harmonics $y_{L_{i} M_{i}}\left(\omega_{i}\right)$.

The $\delta$ function implies that the Bethe-Peierls two-body boundary condition for each two-body interaction can be applied and the third particle can be considered to be far away, i.e.,

$$
\lim _{\rho_{1}^{(k)} \rightarrow 0} \Phi(R ; \Omega)=\left(1-\frac{a^{(k)}}{d_{k} \rho_{1}^{(k)}}\right) y_{L M}\left(\omega_{2}^{(k)}\right) C_{L M}^{(k)} .
$$

Here $y_{L M}$ is a spherical harmonic describing the free space behavior in $\omega_{2}^{(k)}$ and it carries the total angular momentum of the system. The superscript $k$ again indicates the odd-man-out notation. This gives the values of the subhyperangular momentum quantum numbers in the $k$ Jacobi coordinate system as $\lambda_{1}=0$ and $\lambda_{2}=L$, which accounts for the $s$-wave interaction and the total angular momentum $L$. Inserting
Eq. (34) into Eq. (30) gives the hyperangular eigenfunction

$$
\begin{gathered}
\Phi(R ; \Omega)=\frac{2 \mu}{R} \sum_{k} \frac{a^{(k)}}{2 \mu_{k} d_{k}^{3}} N_{L v} C_{L M}^{(k)} y_{L M}\left(\omega_{2}^{(k)}\right) f_{0 L v}^{-}\left(\alpha^{(k)}\right), \\
N_{L v}=\frac{-\Gamma\left(\frac{L-v}{2}\right) \Gamma\left(\frac{L+v+4}{2}\right)}{\sqrt{\pi} \Gamma\left(L+\frac{3}{2}\right)},
\end{gathered}
$$

where $\mu_{k}$ is the two-body reduced mass labeled in the odd-man-out notation and the orthonormality of spherical harmonics has been used to evaluate the $\omega_{1}^{(k) \prime}$ and $\omega_{2}^{(k) \prime}$ integrals. The $\delta$ function in Eq. (24) implies that the integral in $\alpha^{(k) \prime}$ can be accomplished by evaluating at $\alpha_{<}^{(k)}=\alpha^{(k) \prime}=0$.

The analytic equation for the hyperangular eigenfunction in Eq. (35) is not very useful without knowing the hyperangular eigenvalue $v(R)$. To obtain an equation for $v$, the boundary condition given in Eq. (34) must be applied again, i.e.,

$$
\begin{aligned}
y_{L M}\left(\omega_{2}^{\left(k^{\prime}\right)}\right) C_{L M}^{\left(k^{\prime}\right)}= & \lim _{\alpha^{\left(k^{\prime}\right)} \rightarrow 0} \frac{\partial}{\partial \alpha^{\left(k^{\prime}\right)}} \alpha^{\left(k^{\prime}\right)} \Phi\left(R ; \Omega^{\left(k^{\prime}\right)}\right) \\
= & \frac{2 \mu}{R} \sum_{k} \frac{a^{(k)}}{2 \mu_{k} d_{k}^{3}} N_{L \nu} C_{L M}^{(k)} \lim _{\alpha^{\left(k^{\prime}\right)} \rightarrow 0} \frac{\partial}{\partial \alpha^{\left(k^{\prime}\right)}} \alpha^{\left(k^{\prime}\right)} \\
& \times\left[f_{0 L v}^{-}\left(\alpha^{(k)}\right) y_{L M}\left(\omega_{2}^{(k)}\right)\right],
\end{aligned}
$$

To evaluate the limit on the right-hand side of this, we must determine the values of the $k \neq k^{\prime}$ Jacobi coordinates in the limit $\rho_{1}^{\left(k^{\prime}\right)} \rightarrow 0$. Equations (25) and (33) give, for $k \neq k^{\prime}$,

$$
\begin{gathered}
\lim _{\alpha^{\left(k^{\prime}\right) \rightarrow 0}} \alpha^{(k)}=\beta_{k k^{\prime}}=\arctan \left[\frac{\left(m_{1}+m_{2}+m_{3}\right) \mu}{m_{k} m_{k^{\prime}}}\right], \\
\lim _{\alpha^{\left(k^{\prime}\right) \rightarrow 0}} \vec{\rho}_{2}^{(k)} \propto-\vec{\rho}_{2}^{\left(k^{\prime}\right)} .
\end{gathered}
$$

Note that if $f^{-}$is regular at $\beta_{k k^{\prime}}$, then

$$
\lim _{\alpha^{\left(k^{\prime}\right)} \rightarrow 0} \frac{\partial}{\partial \alpha^{\left(k^{\prime}\right)}} \alpha^{\left(k^{\prime}\right)} f_{0 L v}^{-}\left(\alpha^{(k)}\right) \rightarrow f_{0 L v}^{-}\left(\beta_{k k^{\prime}}\right) .
$$

Using this and evaluating the limits in Eq. (36) yields a matrix equation for $C_{L M}^{(k)}$ :

$$
\begin{gathered}
C_{L M}^{\left(k^{\prime}\right)}=\sum_{k} M_{k^{\prime} k}^{L v} C_{L M}^{(k)}, \\
M_{k^{\prime} k}^{L v}= \begin{cases}\frac{2 \mu}{R} \frac{2 \Gamma\left(\frac{L-v}{2}\right) \Gamma\left(\frac{v+L+4}{2}\right)}{\Gamma\left(\frac{L-v-1}{2}\right) \Gamma\left(\frac{L+v+3}{2}\right)} \frac{a^{\left(k^{\prime}\right)}}{2 \mu_{k} d_{k}^{3}} & \text { for } \quad k=k^{\prime}, \\
(-1)^{L} \frac{2 \mu}{R} N_{L v} \frac{a^{(k)}}{2 \mu_{k} d_{k}^{3}} f_{0 L v}^{-}\left(\beta_{k k^{\prime}}\right) & \text { for } \quad k \neq k^{\prime} .\end{cases}
\end{gathered}
$$

The hyperangular eigenvalue $v$ is found by solving the closedform transcendental equation

$$
\operatorname{det}(\mathbf{M}-\mathbf{1})=0
$$

for any given total angular momentum $L$, any set of $s$-wave scattering lengths $a^{(k)}$, and arbitrary masses.

\section{A. Imposing symmetry}

The hyperangular eigenvalues for the general three-body problem with arbitrary exchange symmetry can be found by solving the transcendental equation (41), but the system can be simplified by considering different permutation symmetries and imposing those symmetries on the boundary conditions 
TABLE I. Possible permutation symmetries that may be imposed on the three-body system with $s$-wave interactions are given with the appropriate boundary conditions. B stands for a boson, $\mathrm{F}$ for a fermion, and $\mathrm{X}$ for a distinguishable particle with an arbitrary mass.

\begin{tabular}{llcl}
\hline \hline $\mathrm{X}_{1} \mathrm{X}_{2} \mathrm{X}_{3}$ & $C^{(1)}$ & $C^{(2)}$ & $C^{(3)}$ \\
\hline $\mathrm{BBB}$ & $C$ & $C$ & $C$ \\
$\mathrm{BBX}$ & $C_{1}$ & $C_{1}$ & $C_{2}$ \\
FFX & $C$ & $-C$ & 0 \\
\hline \hline
\end{tabular}

$C_{L M}^{(k)}$. For example, if the particles in question are identical bosons, permutation cannot have any effect on the wave function. Thus, if two particles are exchanged in the two-body subsystem, the boundary condition must remain the same, i.e., $C_{L M}^{(1)}=C_{L M}^{(2)}=C_{L M}^{(3)}=C_{L M}$ and $a^{(1)}=a^{(2)}=a^{(3)}=a$. A complete list of the possible exchange symmetries is given in Table I.

To illustrate this postsymmetrization, we apply the identical boson symmetry with $L=0$ to Eq. (40), resulting in the wellknown transcendental equation for $v$ [4,27-29,31],

$$
\frac{R}{a}=\frac{-3^{1 / 4}\left[\frac{8}{\sqrt{3}} \sin \left(\frac{\pi(v+2)}{6}\right)-(v+2) \cos \left(\frac{\pi(v+2)}{2}\right)\right]}{\sqrt{2} \sin \left(\frac{\pi(v+2)}{2}\right)} .
$$

In the limit where $R / a \rightarrow 0$ the first solution to this transcendental equation gives $(v+2) \rightarrow i s_{0}=1.00624 i$, to six decimal places. Inserting this into Eq. (31) produces a supercritical attractive $1 / R^{2}$ effective potential,

$$
\begin{aligned}
U(R) & =\frac{\hbar^{2}}{2 \mu} \frac{-s_{0}^{2}-1 / 4}{R^{2}}, \\
s_{0} & =1.00624 .
\end{aligned}
$$

This attractive potential is the source of the famous Efimov effect, where an effective attractive dipole-type potential supports an infinite set of three-body bound states that accumulate at the noninteracting three-body threshold, $E=0$.

\section{B. Nonadiabatic couplings}

As in any adiabatic treatment, the effective hyperradial potentials are coupled by nonadiabatic terms that arise from the hyperradial dependence of the hyperangular channel functions. These couplings come in the form of the $P$ and $Q$ matrices in Eq. (5). To find the nonadiabatic coupling matrices, we apply the methods of Ref. [32]. The details of the derivation are shown in the Appendix, the result of which gives the semianalytic expressions for the matrix elements $P_{m n}$ :

$$
\begin{gathered}
P_{m n}=\frac{\sum_{k} C_{m}^{(k)} C_{n}^{(k)} \frac{a^{(k)}}{d_{k} R^{2}}}{\left(\varepsilon_{m}-\varepsilon_{n}\right)} \text { for } n \neq m, \\
-\varepsilon_{n}^{\prime}=\sum_{k}\left(C_{n}^{(k)}\right)^{2} \frac{a^{(k)}}{d_{k} R^{2}} .
\end{gathered}
$$

Here, for notational simplicity, we have set $\varepsilon_{n}=\left(v_{n}+2\right)^{2}$ and all primes indicate a derivative with respect to $R$ (e.g., $\left.\varepsilon_{n}^{\prime}=d \varepsilon_{n} / d R\right)$. Because the hyperangular eigenfunctions are orthonormal, the diagonal part of the $P$ matrix is zero, i.e., $P_{n n}=\frac{1}{2} \frac{\partial}{\partial R}\left\langle\Phi_{n} \mid \Phi_{n}\right\rangle=0$. Equation (45) gives the normaliza- tion condition for $\Phi_{n}$, with an overall phase that is free. This overall phase is chosen here so that $\sum_{k} C_{n}^{(k)}$ is positive. A similar derivation provides the matrix elements $Q_{m n}$ :

$$
\begin{gathered}
Q_{m n}=\delta_{m n}\left(\frac{\varepsilon_{n}^{\prime}+R \varepsilon_{n}^{\prime \prime}+A_{n}}{R^{2} \varepsilon_{n}^{\prime}}+\frac{\varepsilon_{n}^{\prime \prime \prime}}{6 \varepsilon_{n}^{\prime}}\right) \\
+2\left(1-\delta_{m n}\right) \frac{\varepsilon_{n}^{\prime} P_{m n}+B_{m n}}{\left(\varepsilon_{m}-\varepsilon_{n}\right)}, \\
A_{n}=\sum_{k} \frac{a^{(k)}}{d_{k}}\left[\left(C_{n}^{(k)}\right)^{\prime}\right]^{2}, \\
B_{m n}=\sum_{k}\left[C_{m}^{(k)}\left(\frac{a^{(k)}}{d_{k} R^{2}}\right)\left(C_{n}^{(k)}\right)^{\prime}-C_{m}^{(k)} C_{n}^{(k)} \frac{a^{(k)}}{d_{k} R^{3}}\right] .
\end{gathered}
$$

When the symmetries given in Table I are used, there can be a considerable simplification of the expressions for $P_{m n}$ and $Q_{m n}$. For a system of identical bosons where $a^{(1)}=a^{(2)}=$ $a^{(3)}=a, d_{1}=d_{2}=d_{3}=d$, and $C_{n}^{(1)}=C_{n}^{(2)}=C_{n}^{(3)}=C_{n}$, $P_{m n}$ and $Q_{m n}$ are given by

$$
\begin{gathered}
P_{m n}=\frac{\sqrt{\varepsilon_{m}^{\prime} \varepsilon_{n}^{\prime}}}{\left(\varepsilon_{m}-\varepsilon_{n}\right)}, \\
Q_{m n}=\delta_{m n}\left[-\frac{1}{4}\left(\frac{\varepsilon_{n}^{\prime \prime}}{\varepsilon_{n}^{\prime}}\right)^{2}+\frac{1}{6} \frac{\varepsilon_{n}^{\prime \prime \prime}}{\varepsilon_{n}^{\prime}}\right] \\
+\left(1-\delta_{m n}\right)\left[\frac{2 \varepsilon_{n}^{\prime} \sqrt{\varepsilon_{n}^{\prime} \varepsilon_{m}^{\prime}}}{\left(\varepsilon_{m}-\varepsilon_{n}\right)^{2}}-\frac{\varepsilon_{n}^{\prime \prime}}{\left(\varepsilon_{m}-\varepsilon_{n}\right)} \sqrt{\left.\frac{\varepsilon_{m}^{\prime}}{\varepsilon_{n}^{\prime}}\right],}\right.
\end{gathered}
$$

which are in agreement with previously calculated nonadiabatic corrections for the three identical boson system [27].

\section{THREE DISTINGUISHABLE INTERACTING PARTICLES}

In this section the adiabatic three-body potentials and the nonadiabatic couplings are applied to the case of three distinguishable equal-mass particles. This system has been realized, for instance, in ultracold three-component Fermi gases of ${ }^{6} \mathrm{Li}$ atoms $[9,10]$ which has sparked a great deal of recent theoretical interest [33-36]. The scaling behaviors and recombination rates we discuss in this section can be found in Ref. [35]. We derive them here to illustrate the power of the methods presented in this paper. The scattering lengths near the resonance positions used here, as functions of magnetic field, are given in Refs. [9,10,37] by

$$
a^{(k)}=a_{b}\left[1-\frac{\Delta}{B-B_{0}}\right]\left[1+\alpha\left(B-B_{0}\right)\right] ;
$$

for $k=1$ :

$$
\begin{aligned}
a_{b} & =-1450 a_{0}, B_{0}=834.15 \mathrm{G}, \\
\Delta & =300 \mathrm{G}, \quad \text { and } \quad \alpha=4 \times 10^{-4} \mathrm{G}^{-1} ;
\end{aligned}
$$

for $k=2$ :

$$
\begin{aligned}
a_{b} & =-1727 a_{0}, B_{0}=690.4 \mathrm{G} \\
\Delta & =122.2 \mathrm{G}, \quad \text { and } \quad \alpha=2 \times 10^{-4} \mathrm{G}^{-1} ;
\end{aligned}
$$

for $k=3$ :

$$
\begin{aligned}
a_{b} & =-1490 a_{0}, B_{0}=811.22 \mathrm{G}, \\
\Delta & =222.3 \mathrm{G}, \quad \text { and } \quad \alpha=3.95 \times 10^{-4} \mathrm{G}^{-1} ;
\end{aligned}
$$




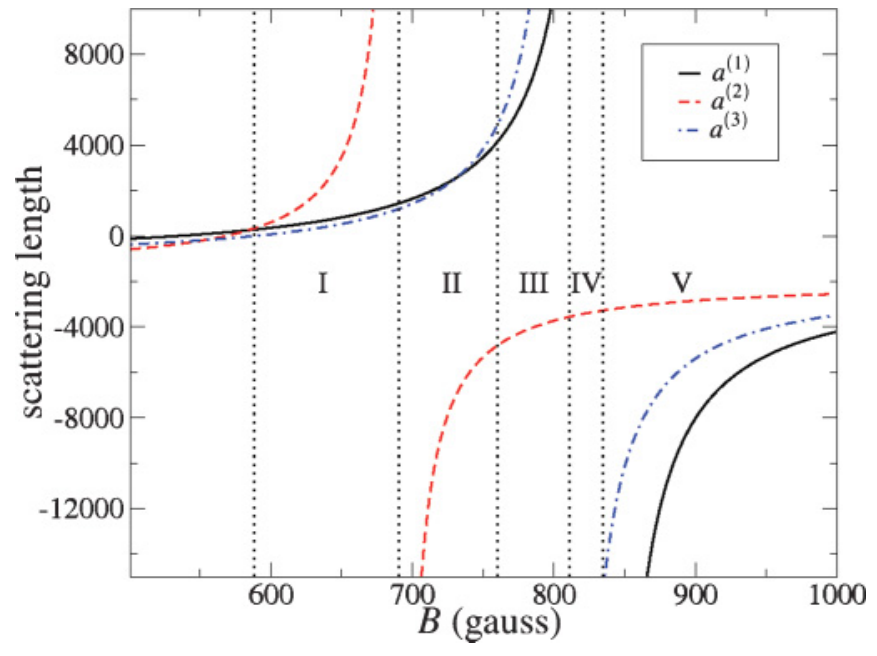

FIG. 1. (Color online) All possible $s$-wave scattering lengths are shown for the lowest three Zeeman states of $\mathrm{Li}^{6}$ from Ref. [37]. Each marked region gives a different set of length scale discrepancies. Here $a^{(k)}$ is the scattering length between two atoms in states $|i\rangle$ and $|j\rangle$ with $k$ as the component not involved in the interaction.

where $a_{0}$ is the Bohr radius. The Fano-Feshbach resonances in this system allow for a large variety of tunable interactions.

This series of overlapping resonances produces five different regions of magnetic field, shown in Fig. 1, near the three resonance positions, each possessing a distinct behavior. In all five regions, the scattering lengths are much larger than the effective range, allowing for the use of the zero-range interaction assumptions. Table II shows the various length scale disparities in these regions.

Figure 2(a) shows an example of the lowest four hyperangular eigenvalues $(v+2)^{2}$ obtained from solving Eq. (40) for $a^{(1)}=a^{(3)}$ and $a^{(2)}=1000 a^{(1)}$. This is provided as an example that is qualitatively similar to the behavior of the system in region $\mathrm{I}$. When the hyperradius is in a region where all other length scales are much different, the hyperangular eigenvalue $(v+2)^{2}$ becomes constant, or, in the case of two-body bound states, becomes proportional to $R^{2}$. This behavior can be interpreted as giving a universal set of potential curves from Eq. (31). For example, in region I, where $r_{0} \ll a^{(3)} \lesssim a^{(1)} \ll$ $a^{(2)}$, there are three hyperradial regions: $r_{0} \ll R \ll a^{(3)} \lesssim$ $a^{(1)} \ll a^{(2)} ; r_{0} \ll a^{(3)} \lesssim a^{(1)} \ll R \ll a^{(2)} ;$ and $r_{0} \ll a^{(3)} \lesssim$ $a^{(1)} \ll a^{(2)} \ll R$. In each region the hyperangular eigenvalues take on the universal value that is expected for resonant interactions $[2,27,28]$.

TABLE II. Possible tunable interaction regimes near the resonances of ${ }^{6} \mathrm{Li}$.

\section{Region}

\begin{tabular}{lll}
\hline I & $r_{0} \ll a^{(3)} \lesssim a^{(1)} \ll a^{(2)}$ & $a^{(1)}, a^{(2)}, a^{(3)}>0$ \\
II & $r_{0} \ll a^{(3)} \sim a^{(1)} \ll\left|a^{(2)}\right|$ & $a^{(2)}<0 ; a^{(1)}, a^{(3)}>0$ \\
III & $r_{0} \ll\left|a^{(2)}\right| \ll a^{(1)}, a^{(3)}$ & $a^{(2)}<0 ; a^{(1)}, a^{(3)}>0$ \\
IV & $r_{0} \ll\left|a^{(2)}\right| \ll\left|a^{(1)}\right|, a^{(3)}$ & $a^{(2)}, a^{(1)}<0 ; a^{(3)}>0$ \\
V & $r_{0} \ll\left|a^{(2)}\right| \ll\left|a^{(1)}\right| \ll\left|a^{(3)}\right|$ & $a^{(1)}, a^{(2)}, a^{(3)}<0$ \\
\hline
\end{tabular}

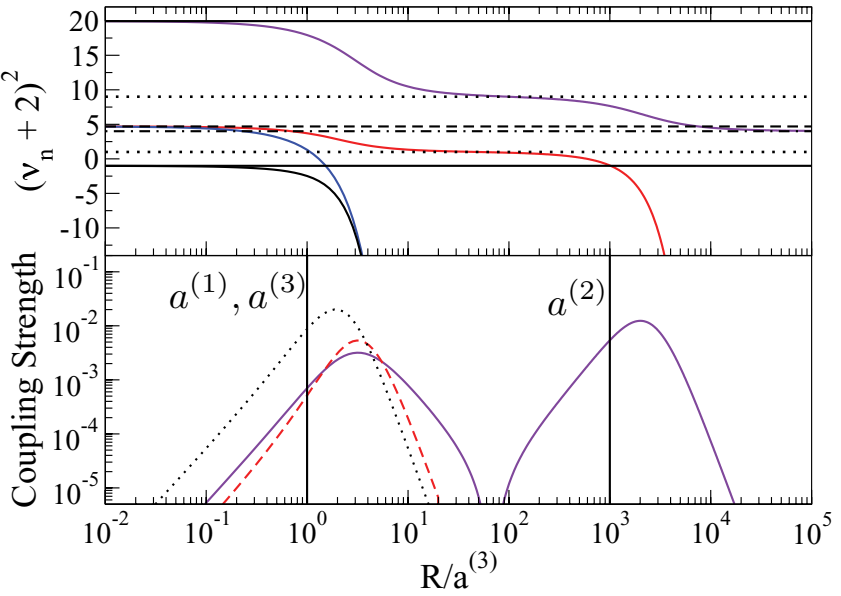

FIG. 2. (Color online) (a) For an example system having $a^{(1)}=$ $a^{(3)}$ and $a^{(2)}=1000 a^{(1)}$, the first four hyperangular eigenvalues are shown as functions of the hyperradius. The solid black horizontal lines show the expected behavior for three identical resonantly interacting bosons. The dashed line gives the behavior of two identical fermions interacting resonantly with a third distinguishable particle. Dotted lines give the expected universal behavior for a single resonant scattering length. Finally, the dot-dashed line is the lowest expected free space eigenvalue for three distinguishable free particles. (b) The coupling strengths between the third and fourth (purple solid curve), the first and fourth (red dashed curve), and the first and third (black dotted curve) adiabatic potentials are shown as a function of $R$.

Figure 3 schematically shows the behavior of the first few hyperradial effective potentials from Eq. (31). The grey areas are the regions where potentials are transitioning from one universal behavior to the next. The zero-range pseudopotential cannot describe the short-range details of the interaction, meaning that the potentials found here are only valid for $R \gg$ $r_{0}$, where $r_{0}$ is a short-range parameter shown schematically as the labeled blue region on the left of Fig. 3.

Figure 2(b) shows the coupling strength, $P_{m n}^{2} /$ $2 \mu\left[u_{m}(R)-u_{n}(R)\right]$, between the different potentials. The

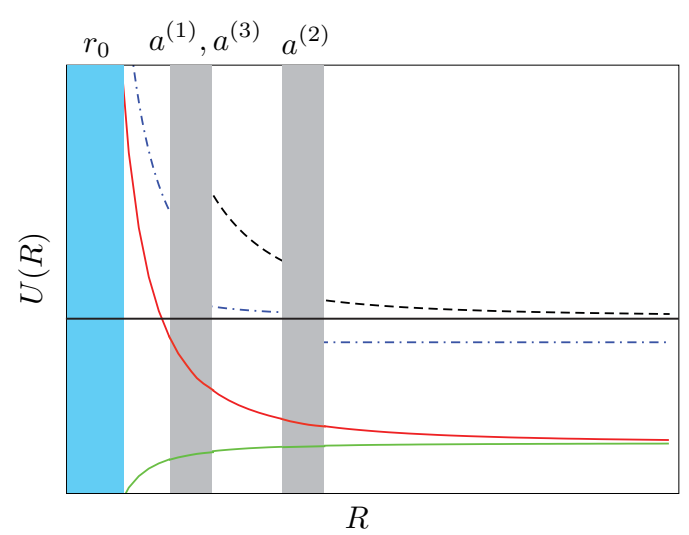

FIG. 3. (Color online) A schematic picture of the first four hyperradial potentials in region I. The grey areas, labeled by the appropriate scattering lengths, indicate regions where the potentials are changing from one universal behavior to another. The blue region on the left, labeled by $r_{0}$, indicates the short-range region where the zero-range pseudopotential can no longer be applied. 

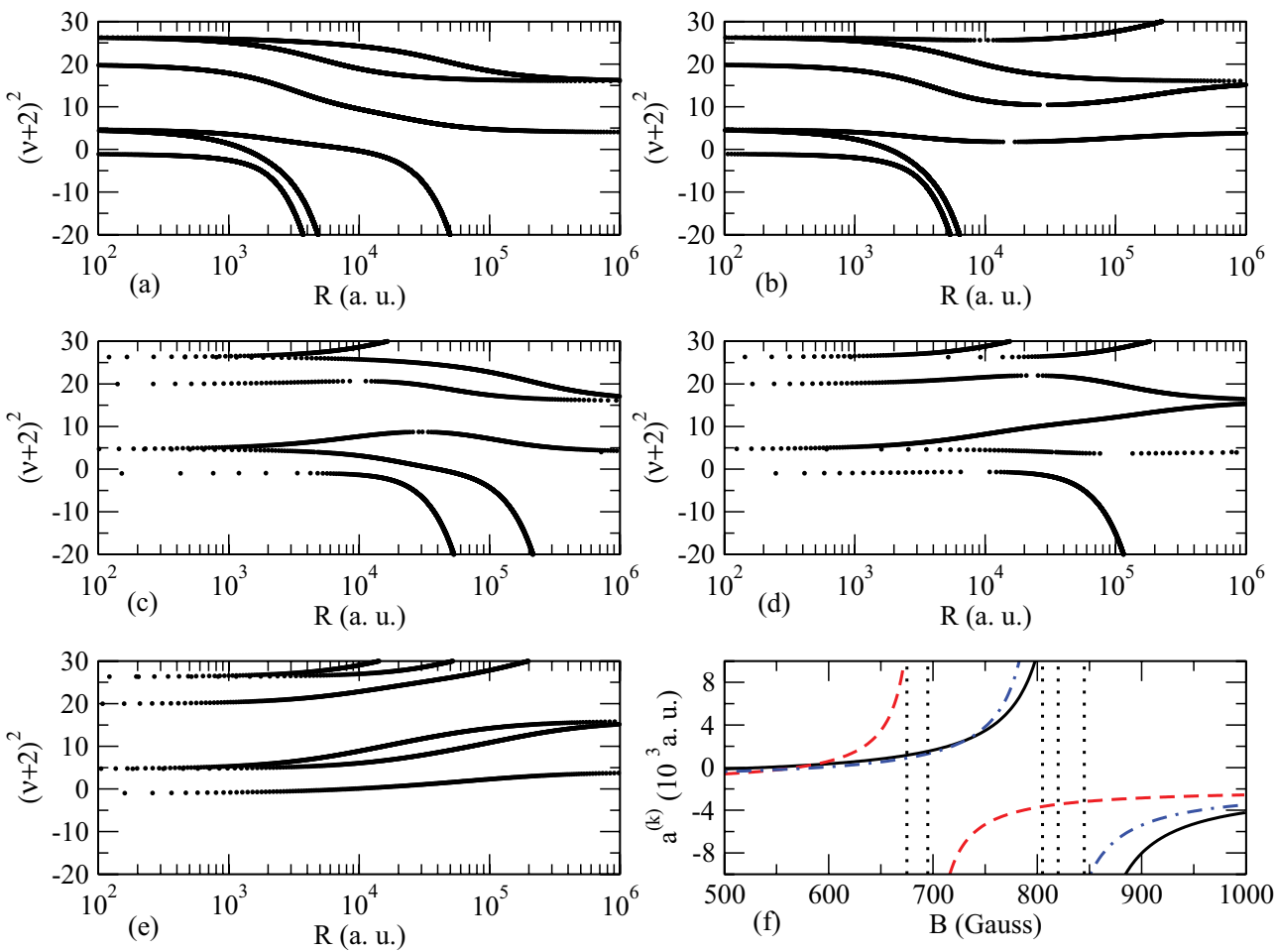

FIG. 4. (Color online) (a)-(e) Examples of the hyperangular eigenvalues from each region of magnetic field as a function of the hyperradius in atomic units. (f) The three $s$-wave scattering lengths shown as a reference vs the magnetic field strength. The vertical dotted lines, from right to left, show the magnetic fields at which the hyperangular eigenvalues in (a)-(e) were found, $B=675,695,805,820$, and $845 \mathrm{G}$, respectively.

places where this coupling peaks are the points where a transition between curves is the most probable. Figures 4(a)4(e) are examples of the hyperangular eigenvalues found in each region. The magnetic field at which each set of eigenvalues are found is shown as dotted lines in Fig. 4(d) from left to right for Figs. 4(a)-4(e), respectively. In each figure the hyperangular eigenvalue can be seen flattening out to a universal constant in each region of length scale discrepancy. As the magnetic field is scanned through each resonance, one two-body bound state becomes a virtual state. This behavior can be seen in the hyperangular eigenvalues that diverge toward $-\infty$. As each resonance is crossed, one of the hyperangular eigenvalue curves goes from diverging to $-\infty$ to converging to $(v+2)^{2} \rightarrow 4$.

As a final examination of this system, we extract the scaling of the low-energy three-body recombination rate, i.e., the rate at which three particles collide and form a dimer and a free particle. The lowest three-body curve, the lowest potential that goes to the three-free-particle threshold, is the potential that dominates this process. Contributions from higher hyperradial potentials will be suppressed due to larger tunneling barriers. One limitation of the zero-range pseudopotential is that it only admits at most one dimer of each type. The process of three-body recombination releases the binding energy of the dimer state as kinetic energy between the dimer and remaining particle.

The event rate coefficient for $N$ initially unbound particles with total orbital angular momentum $L$ to make a transition from a hyperspherical potential curve with hyperangular eigenvalue $\lambda$ to a lower lying final state is given by $[4,38]$

$$
K_{N}=\frac{\hbar k}{\mu} N_{S}\left(\frac{2 \pi}{k}\right)^{d-1} \frac{\Gamma\left(\frac{d}{2}\right)}{2 \pi^{d / 2}} \sum_{i, f}(2 L+1)\left|T_{f i}\right|^{2}
$$

where $d$ is the total dimension of the system (in the case of three-body recombination $d=6$ ), $T_{f i} \equiv S_{f i}-\delta_{f i}$ is the transition matrix element between an initial three-body entrance channel $i$ and a final exit channel $f$, and $k=\sqrt{2 \mu E} / \hbar$ is the wave number of the asymptotic hyperradial wave function. The sum in this equation runs over all the initial, asymptotic channels with total angular momentum $L$ that contribute to the scattering process. In Eq. (49), $N_{S}$ is the number of permutational symmetries in the system. For three distinguishable particles $N_{S}=1$, but it can be different; for instance, for $N$ identical bosons, $N_{S}=N$ !. For the purposes of this study we will concentrate on the three-body recombination processes that result in trap loss processes, where the energy released in the recombination can be assumed sufficient to eject the remaining fragments from a trap. In this section we assume that the confined gas is at low enough densities and temperatures to remain in the threshold regime, i.e., $1 / k \gg\left|a_{l}\right|$ where $a_{l}$ is the largest of the three scattering lengths from Fig. 1 at any given magnetic field. With this assumption the presence of an external trapping potential plays no significant role in the scaling behavior of three-body recombination.

In the low-energy regime, only the lowest $L=0$ initial three-body channel will contribute, while higher channels will be suppressed. The sum over final $T$ matrix elements can be 
approximated using the Wentzel-Kramers-Brillouin (WKB) phase in the entrance channel $[28,38]$ :

$$
\sum_{f}\left|T_{f i}\right|^{2} \approx \frac{e^{-2 \gamma}}{2} \frac{\sinh 2 \eta}{\cos ^{2} \phi+\sinh ^{2} \eta},
$$

where $\eta$ is an imaginary phase which parametrizes the losses from the incoming channel. In Eq. (50), $\gamma$ is the total WKB tunneling integral between the outer classical turning point and the hyperradial position at which the transition to the outgoing state occurs, i.e.,

$$
\gamma=\operatorname{Re}\left[\int_{R_{0}}^{R_{T}} \sqrt{\frac{2 \mu}{\hbar^{2}}[U(R)-E]+\frac{1}{4 R^{2}}} d R\right],
$$

where $E$ is the initial three-body energy, $R_{T}$ is the outer classical turning point, and $R_{0}$ is the position at which the coupling between the incoming and outgoing channels peaks. In Eq. (50), $\phi$ is the WKB phase accumulated in any inner attractive well:

$$
\phi=\operatorname{Im}\left[\int_{R_{0}}^{R_{T}} \sqrt{\frac{2 \mu}{\hbar^{2}}[U(R)-E]+\frac{1}{4 R^{2}}} d R\right] .
$$

The extra repulsive $1 / 4 R^{2}$ term in Eqs. (51) and (52) is due to the Langer correction [39]. The total $T$-matrix element will depend on the detailed nature of the real short-range interactions and the behavior of the outgoing channels, but the scaling behavior with the scattering lengths will be determined by Eq. (50). In each region of magnetic field, there are different length scale discrepancies and different numbers of bound states. As a result, we will examine each region separately.

$$
\text { 1. Region I }\left(a^{(1)} \sim a^{(3)} \ll a^{(2)}\right)
$$

Figure 4(a) shows the behavior of the first few hyperangular eigenvalues in region I. The first three eigenvalues correspond to dimer states, while the fourth corresponds to the lowest three-body potential and is the entrance channel that will control three-body recombination. The lower two dimer states are relatively deeply bound with binding energies, $\hbar^{2} / m a^{2}$, on the order of $10^{-12}$ Hartree. This is comparable to the trap depth energy of a normal magneto-optical trap for experiments with ${ }^{6} \mathrm{Li}[9,10]$, meaning that recombination into these dimer channels typically releases enough energy to eject the remaining dimer-atom system from the trap.

In the limit where $R \gg a^{(3)}$, the three atoms are far enough apart to be in the noninteracting regime. This means that the hyperangular eigenfunction limits to the lowest allowed three-body hyperspherical harmonic with its corresponding eigenvalue, $(v+2)^{2} \rightarrow 4$. In this limit the hyperradial potential becomes

$$
U\left(R \gg a^{(3)}\right)=\frac{\hbar^{2}}{2 \mu} \frac{4-1 / 4}{R^{2}} .
$$

For very low energy scattering, the classical turning point in Eq. (50) is approximately

$$
R_{T}=\frac{1}{2 k} .
$$

In fact, this will be the turning point for all of the three-body recombination processes discussed in this section.

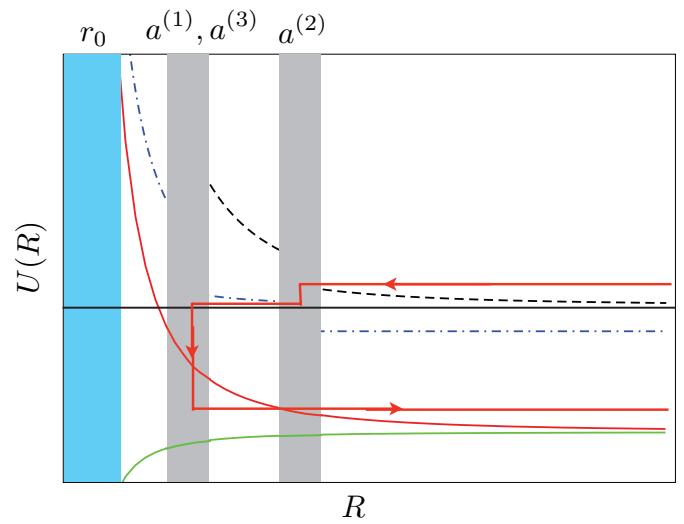

FIG. 5. (Color online) A schematic of the path for three-body recombination in region I. Transition regions are labeled by the appropriate length scale, and the short-range nonuniversal region is labeled by $r_{0}$.

It is possible for recombination to occur directly between the lowest three-body curve and the deep dimer channels, but this direct process is strongly suppressed due to the large tunneling barrier in the three-body potential at small $R$. The favored path is through a transition to the weakly bound dimer channel, shown schematically in Fig. 5. The coupling between the lowest three-body channel and the weakly bound dimer channel peaks at approximately $R \sim a^{(2)}$, while the coupling peak between the weakly bound dimer channel and the remaining two dimer channels occurs at approximately $R \sim a^{(3)} \sim a^{(1)}$. In the regime where $a^{(1)} \sim a^{(3)} \ll R \ll a^{(2)}$ the three particles are so far apart that they cannot see the smaller scattering lengths $a^{(1)}$ and $a^{(3)}$, but the third scattering length is so large compared to the hyperradius that it might as well be infinite. This leads to a universal potential whose hyperangular eigenvalue can be found by solving Eq. (40) with $a^{(1)}=a^{(3)}=0$ and $a^{(2)} \rightarrow \infty$, i.e.,

$$
\begin{array}{r}
U(R)=\frac{\hbar^{2}}{2 \mu} \frac{p_{1}^{2}-1 / 4}{R^{2}}, \\
p_{1}=(v+2)=1 .
\end{array}
$$

This intermediate universal behavior can clearly be seen in Fig. 2(a).

The behavior of each channel can be approximated by the universal behavior of the hyperradial potential in each region. Under this assumption, using Eq. (50), the tunneling probability is given by

$$
\begin{gathered}
e^{-2 \gamma} \propto \exp \left[-2 \int_{a^{(3)}}^{a^{(2)}} d R \sqrt{\frac{p_{1}^{2}}{R^{2}}-\frac{2 \mu}{\hbar^{2}} E}\right. \\
\left.-2 \int_{a^{(2)}}^{R_{T}} d R \sqrt{\frac{4}{R^{2}}-\frac{2 \mu}{\hbar^{2}} E}\right] .
\end{gathered}
$$

If the scattering energy is very small, $E \ll \hbar^{2} / m\left[a^{(2)}\right]^{2}$, then the energy dependence in these integrands becomes negligible leaving,

$$
e^{-2 \gamma} \propto k^{4}\left(a^{(2)} a^{(3)}\right)^{2} .
$$


Inserting this in for the $T$-matrix element in Eq. (49) gives the scaling behavior of the recombination rate with the scattering lengths [28]:

$$
K_{3} \propto\left(a^{(2)} a^{(3)}\right)^{2} .
$$

It was assumed here the final transition occurs at $R \sim a^{(3)}$ leading to the scaling behavior with $a^{(3)}$, but the transition could just as easily have occurred at $R \sim a^{(1)}$. $a^{(1)}$ and $a^{(3)}$ are approximately equal here, and which one dominates the transition depends on the short-range behavior of the real twobody interaction. To extract the scaling behavior with respect to $a^{(1)}$, one can simply replace $a^{(3)}$ with $a^{(1)}$ in Eq. (58) as long as $a^{(1)}$ and $a^{(3)}$ are approximately equal.

\section{Region II $\left(a^{(1)} \sim a^{(3)} \ll\left|a^{(2)}\right|\right)$}

The recombination in region II is simpler than in region I, as there is no weakly bound intermediate state. Again, we assume that the trap loss recombination is dominated by transitions to the two remaining dimer states seen in Fig. 4(b). The lowest three-body potential has a coupling to these channels that peaks at $R \sim a^{(1)}$ and $R \sim a^{(3)}$. For $R \gg\left|a^{(2)}\right|$ the hyperangular eigenvalue takes on the noninteracting value $(v+2) \rightarrow 2$. For $a^{(1)}, a^{(3)} \ll R \ll\left|a^{(2)}\right|$ the universal hyperangular eigenvalue $(v+2)=p_{1}=1$ is seen again [27-29]. Ignoring the transitional region between these two regimes, the transition probability is given by

$$
e^{-2 \gamma} \propto \exp \left[-2\left(\int_{a^{(3)}}^{\left|a^{(2)}\right|} d R \sqrt{\frac{p_{1}^{2}}{R^{2}}}+\int_{\left|a^{(2)}\right|}^{R_{T}} d R \sqrt{\frac{4}{R^{2}}}\right)\right] .
$$

Inserting this into Eq. (49) gives a recombination rate that has the same scaling behavior as in region I [28]:

$$
K_{3} \propto\left(a^{(2)} a^{(3)}\right)^{2} .
$$

Again it is assumed that the final transition occurs at $R \sim a^{(3)}$, but it could occur at $a^{(1)}$ as well. As in region I, the scaling behavior with respect to $a^{(1)}$ can be found by simply replacing $a^{(3)}$ with $a^{(1)}$ in Eq. (58) as long as $a^{(1)}$ and $a^{(3)}$ are close.

$$
\begin{gathered}
\text { 3. Region III }\left(\left|a^{(2)}\right| \ll a^{(1)} \sim a^{(3)}\right) \text { and region IV } \\
\left(\left|a^{(2)}\right| \ll\left|a^{(1)}\right| \sim a^{(3)}\right)
\end{gathered}
$$

In region III, none of the dimers predicted by the zero-range model have enough binding energy to cause trap loss. While recombination can occur into these channels, we will focus on the process of recombination to deeply bound states here. In reality, the deep interaction potential between two Li atoms in different spin states admits many deeply bound dimer states, and a true hyperspherical description of the system would have channels going to each possible dimer-atom threshold. The energy released in recombining into these deep states is enough to kick the atoms out of any normal trap. Because the deeply bound states are of the size of the range of the interaction, coupling to the deeply bound hyperradial channels will peak at small hyperradius, $R \sim r_{0}$, and the rate can be found by studying the tunneling probability of reaching these states.

As with the recombination process in region I, the most favorable pathway involves multiple steps. Starting from the lowest three-body channel, a transition is made to either the

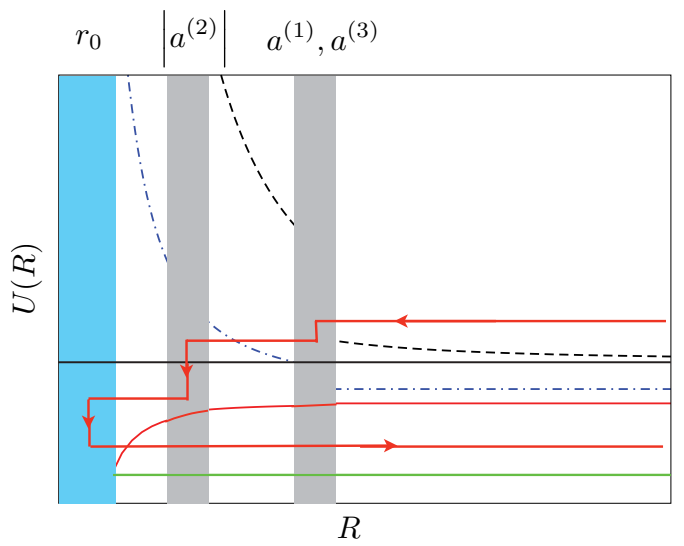

FIG. 6. (Color online) A schematic of the potentials and the path for three-body recombination in region III. Again the labeled grey regions indicate a transition from one universal potential behavior to another. The lowest (green) line represents the hyperradial potential for a deeply bound dimer state. The blue area on the left labeled by $r_{0}$ is the short-range region not described by zero-range interactions.

first or second weakly bound dimer channel. Because $a^{(1)}$ and $a^{(3)}$ are similar in magnitude, the coupling to these channels peaks in the same region. If the transition is made to the highest dimer channel, then another transition is made directly to the second.

This pathway is shown schematically in Fig. 6. An interesting thing occurs in the lowest weakly bound potential when $\left|a^{(2)}\right| \ll R \ll a^{(1)} \sim a^{(3)}$ : the universal potential becomes attractive. This region of attractive potential gives rise to a number of phenomena. For instance, in the limit $a^{(1)}, a^{(3)} \rightarrow$ $\infty$, the universal attractive potential supports an infinite number of geometrically spaced three-body bound states, giving rise to the Efimov effect. In the process of three-body recombination to deeply bound dimer states, though, there is no tunneling suppression in this channel, and the hyperradial wave function merely accumulates phase in this region. As a result, the WKB tunneling probability is controlled by the transition at $R \sim a^{(1)}, a^{(3)}$ :

$$
e^{-2 \gamma} \propto \exp \left[-2 \int_{a^{(1)}}^{R_{T}} d R \sqrt{\frac{4}{R^{2}}}\right] .
$$

Inserting this into Eq. (49) gives the scaling of three-body recombination to deep dimer states as

$$
K_{3} \propto\left[a^{(1)}\right]^{4} .
$$

Again, it is assumed here that $a^{(1)}$ and $a^{(3)}$ are similar in magnitude. If this is not the case, for instance, if $a^{(1)} \gg a^{(3)}$, then a scaling behavior similar to that of Eq. (58) is recovered:

$$
K_{3} \propto\left[a^{(1)} a^{(3)}\right]^{2} .
$$

In Region IV there is only a single weekly bound dimer state available, and trap loss will occur through recombination to deeply bound dimers. The path here is similar to that of Region III, where a transition happens from the lowest three-body channel to the weakly bound dimer channel. From there the hyperradial wave function can go to the small $R$ region 


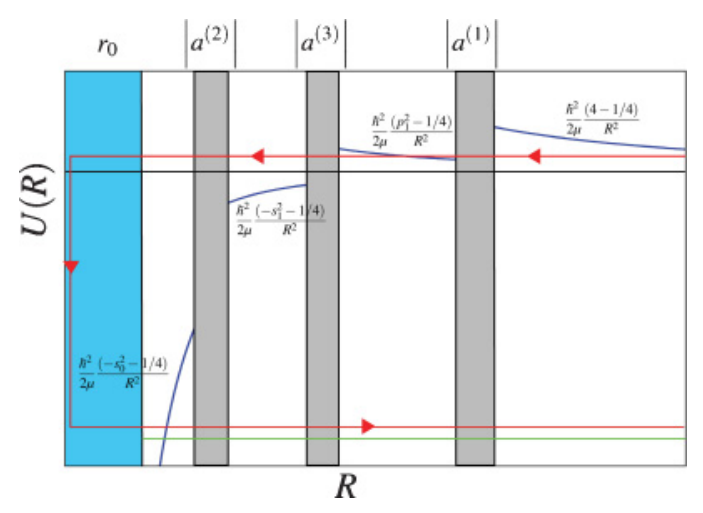

FIG. 7. (Color online) A schematic of the lowest hyperradial potential with the path for three-body recombination to deeply bound states. The lowest (green) line represents the hyperradial potential for a deeply bound dimer state. Labeled grey areas indicate transition regions from one universal behavior to another, and the far-left blue region indicates the short-range regime.

without further suppression. This process then yields the same three-body recombination scaling behavior as Eq. (62) when $a^{(1)} \sim\left|a^{(3)}\right|$. When $a^{(3)} \gg\left|a^{(1)}\right|$, the scaling predicted by Eq. (63) is recovered.

\section{Region $V\left(\left|a^{(2)}\right| \ll\left|a^{(3)}\right| \ll\left|a^{(1)}\right|\right)$}

In this regime the recombination process is entirely controlled by the lowest three-body channel, shown schematically in Fig. 7. The hyperradial potential has three universal regimes. The first, when $r_{0} \ll R \ll\left|a^{(2)}\right| \ll\left|a^{(3)}\right| \ll\left|a^{(1)}\right|$, is identical to that of three strongly interacting bosons. The hyperangular eigenvalue $(v+2)^{2}$ is the first solution to Eq. (42) in the limit where $R / a \rightarrow 0$, yielding the hyperradial potential,

$$
\begin{gathered}
U(R)=\frac{\hbar^{2}}{2 \mu} \frac{-\left(s_{0}\right)^{2}-1 / 4}{R^{2}}, \\
s_{0}=1.0062 .
\end{gathered}
$$

In the next regime, when $r_{0} \ll\left|a^{(2)}\right| \ll R \ll\left|a^{(3)}\right| \ll\left|a^{(1)}\right|$, the three particles are far enough apart so as not to see the smallest scattering length. As a result the hyperangular eigenvalue is governed by Eq. (40) with the BBX symmetry of Table I imposed:

$$
\begin{gathered}
U(R)=\frac{\hbar^{2}}{2 \mu} \frac{-\left(s_{1}\right)^{2}-1 / 4}{R^{2}}, \\
s_{1}=0.4137
\end{gathered}
$$

In the regime where $r_{0} \ll\left|a^{(2)}\right| \ll\left|a^{(3)}\right| \ll R \ll\left|a^{(1)}\right|$, there is only one scattering length seen by the system, and the universal potential becomes that of Eq. (55). In the final regime, where the hyperradius is much larger than all of the scattering lengths, the potential goes to the noninteracting behavior of a hyperspherical harmonic.

The transition to a deeply bound dimer state occurs at $R \sim r_{0}$ following the path shown in Fig. 7. To get to this region, the wave function must first tunnel through a barrier, leading to suppression of the recombination rate. Once through the barrier, the wave function accumulates phase in the attractive potential regime. If enough phase can be accumulated in this regime, then a three-body bound state (a so-called Efimov state) can be present leading to a resonance in the recombination rate. The final recombination rate for this process is $[4,6,27,28]$

$$
K_{3} \propto A \frac{\sinh 2 \eta}{\cos ^{2}\left(\phi_{\mathrm{WKB}}\right)+\sinh ^{2} \eta},
$$

where $\eta$ is controlled by the short-range properties of the system, $\phi_{\mathrm{WKB}}$ is the WKB phase accumulated in the attractive regime from $r_{0}$ to $\left|a^{(3)}\right|$, and $A$ is proportional to the tunneling suppression through the barrier:

$$
\begin{gathered}
A \propto\left[a^{(3)} a^{(1)}\right]^{2}, \\
\phi_{\mathrm{WKB}}=s_{1} \ln \left(\frac{a^{(3)}}{a^{(2)}}\right)+s_{0} \ln \left(\frac{\left|a^{(2)}\right|}{r_{0}}\right) .
\end{gathered}
$$

Figure 8(a) shows the log of the three-body recombination rate in arbitrary units as a function of the magnetic field with $\eta=0.001$. The short-range length scale here is chosen to be approximately the van der Waals length of ${ }^{6} \mathrm{Li}, r_{0}=r_{d} \approx$ 30 atomic units. Figure 8(b) shows the scattering lengths in the same region of magnetic fields for reference. An Efimov resonance can clearly be seen at $B=942 \mathrm{G}$ when $\phi_{\mathrm{WKB}}=$ $3 \pi / 2$. This is in rough agreement with the predicted position of $B=1160 \mathrm{G}$ found in Ref. [33]. The exact position of this resonance is somewhat sensitive to the short-range length scale $r_{0}$ which should be fit to experimental data. We have chosen $r_{0}$ as the Van der Waals length here for illustrative purposes. A WKB phase that is $3 \pi / 2$ at the resonance indicates that this corresponds to the second Efimov state intersecting the continuum. The first Efimov state remains bound throughout this region. Because $a^{(1)}$ becomes resonantly large as $B \rightarrow$ $834.15 \mathrm{G}$, the $\left[a^{(3)}\right]^{2}$ scaling from Eq. (67) gives the large recombination rate seen in the lower field region of Fig. 8(a).

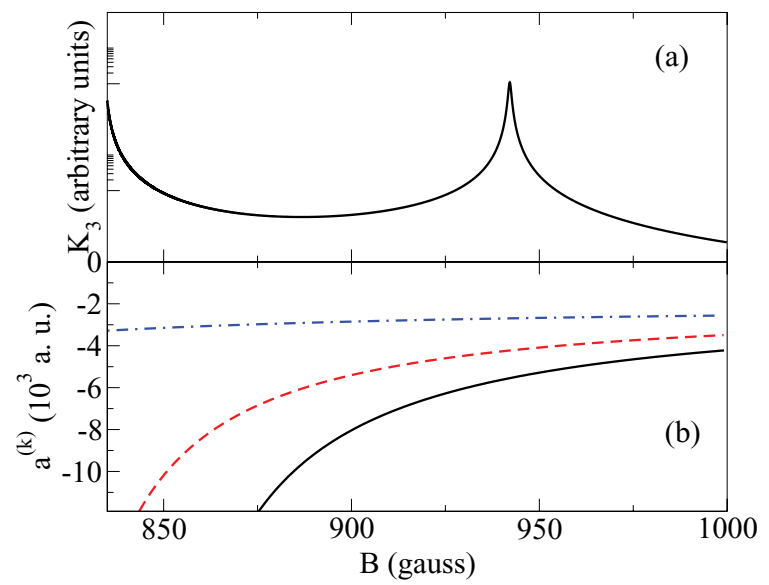

FIG. 8. (Color online) (a) The three-body recombination rate from Eq. (66) for ${ }^{6} \mathrm{Li}$ in arbitrary units as a function of magnetic field with $\eta=0.001$ and the short-range length scale chosen to be approximately the van der Waals length, $r_{0}=r_{d} \approx 30$ a.u. The large $y$-axis tick marks indicate orders of magnitude. (b) The three scattering lengths $a^{(1)}$ (solid black curve), $a^{(2)}$ (dashed red curve), and $a^{(3)}$ (dot-dashed blue curve) in atomic units as a function of magnetic field in region $\mathrm{V}$. 
With three overlapping resonances, ${ }^{6} \mathrm{Li}$ provides a rich hunting ground for the study of three-body physics. Further, because it is a fermionic atom, three-body interactions involving only two of the three lowest components are strongly suppressed meaning that the majority of the three-body physics is controlled by a system of three distinguishable particles. While only the processes of three-body recombination that lead to trap losses were studied in this section, there is still a rich and complex array of behaviors not discussed that can be described using the model presented here.

\section{SUMMARY}

In this work we have developed a form for the hyperangular Green's function in arbitrary dimensions. The derivation of the Green's function is simple and follows easily from a standard Sturm-Liouville problem. By dividing a $d$-dimensional space into physically meaningful subspaces, this Green's function avoids the slow convergence often seen in a spectral expansions form, while maintaining a physically intuitive set of hyperangular coordinates.

We have also used the hyperangular Green's function to solve the three-body problem with zero-range $s$-wave interaction for arbitrary scattering lengths, particle masses, and total angular momentum. With simple root finding, the adiabatic hyperangular channel functions and adiabatic potentials can be extracted. The resulting transcendental equation is in exact agreement with that derived using Faddeev-like decompositions. To complete the problem, we have also derived general expressions for the nonadiabatic corrections to the potentials that are analytic up to root finding.

The results of the general three-body problem were then applied to the three lowest hyperfine components of ${ }^{6} \mathrm{Li}$ near a set of overlapping resonances. By a simple WKB formalism, the scaling behavior of the rate constant for trap loss three-body recombination events was extracted throughout the overlapping two-body resonances. Signatures of an Efimov style resonance are also predicted to appear at high field strengths. Throughout the resonances, all of the scattering lengths are very large compared to the length scale of the two-body interaction, indicating that the results presented here are universal. The simple and intuitive nature of the Lippmann-Schwinger equation in the three-body problem indicates that this Green's function based method may be applicable in the context of the four-body problem, but this extension is the subject of ongoing inquiry.

\section{ACKNOWLEDGMENTS}

The authors would like to thank D. Blume and J. P. D'Incao for many useful discussions. This research was supported in part by funding from the National Science Foundation. S. T. R. acknowledges support from a NSF grant to ITAMP at Harvard University and the Smithsonian Astrophysical Observatory.

\section{APPENDIX}

In this Appendix we sketch the derivation of the formulas for the nonadiabatic $P$ and $Q$ matrix elements given in Eqs. (44) and (46). We begin by considering matrix elements dealing with the derivative of the adiabatic Schrödinger equation:

$$
\begin{gathered}
\left\langle\Phi_{n}^{\prime}\left|\left(\Lambda^{2}-\varepsilon_{m}\right)\right| \Phi_{m}\right\rangle=0, \\
-\varepsilon_{n}^{\prime}\left\langle\Phi_{m} \mid \Phi_{n}\right\rangle+\left\langle\Phi_{m}\left|\left(\Lambda^{2}-\varepsilon_{n}\right)\right| \Phi_{n}^{\prime}\right\rangle=0,
\end{gathered}
$$

where $\varepsilon_{n}=v_{n}\left(v_{n}+4\right)$ is the hyperangular eigenvalue of the $n$th adiabatic eigenfunction, and the prime indicates a hyperradial derivative has been taken. Taking the difference of these leads to an equation for the nonadiabatic coupling matrix element $P_{m n}$ for $m \neq n$ :

$$
\left\langle\Phi_{n}^{\prime}\left|\Lambda^{2}\right| \Phi_{m}\right\rangle-\left\langle\Phi_{m}\left|\Lambda^{2}\right| \Phi_{n}^{\prime}\right\rangle-\left(\varepsilon_{m}-\varepsilon_{n}\right) P_{m n}+\delta_{m n} \varepsilon_{n}^{\prime}=0 .
$$

The difference $\left\langle\Phi_{n}^{\prime}\left|\Lambda^{2}\right| \Phi_{m}\right\rangle-\left\langle\Phi_{m}\left|\Lambda^{2}\right| \Phi_{n}^{\prime}\right\rangle$ is given by the boundary conditions of the wave functions $\Phi_{m}$ and $\Phi_{n}$ at the coalescence points:

$$
\begin{aligned}
& \left\langle\Phi_{n}^{\prime}\left|\Lambda^{2}\right| \Phi_{m}\right\rangle-\left\langle\Phi_{m}\left|\Lambda^{2}\right| \Phi_{n}^{\prime}\right\rangle \\
& \quad=\sum_{k}\left[\frac{a^{(k)}}{d_{k} R} C_{m} \frac{\partial}{\partial R} C_{n}^{(k)}-C_{m}^{(k)} \frac{\partial}{\partial R}\left(\frac{a^{(k)}}{d_{k} R} C_{n}^{(k)}\right)\right] \\
& \quad=\sum_{k} C_{m}^{(k)} C_{n}^{(k)} \frac{a^{(k)}}{d_{k} R^{2}} .
\end{aligned}
$$

Here the $L M$ subscripts in the boundary values $C_{L M}^{(k)}$ have been suppressed. Inserting Eq. (A3) into Eq. (A2) yields Eq. (44),

$$
\begin{gathered}
P_{m n}=\frac{\sum_{k} C_{m}^{(k)} C_{n}^{(k)} \frac{a^{(k)}}{d_{k} R^{2}}}{\left(\varepsilon_{m}-\varepsilon_{n}\right)} \quad \text { for } n \neq m, \\
-\varepsilon_{n}^{\prime}=\sum_{k}\left(C_{n}^{(k)}\right)^{2} \frac{a^{(k)}}{d_{k} R^{2}} .
\end{gathered}
$$

A similar derivation provides the matrix elements $Q_{m n}$ given in Eq. (46).
[1] V. Efimov, Yad. Fiz. 12, 1080 (1970) [Sov. J. Nucl. Phys. 12, 589 (1971)].

[2] V. N. Efimov, Nucl. Phys. A 210, 157 (1973).

[3] J. H. Macek, Z. Phys. D 3, 31 (1986).

[4] B. D. Esry, C. H. Greene, and J. P. Burke, Phys. Rev. Lett. 83, 1751 (1999).

[5] E. Nielsen and J. H. Macek, Phys. Rev. Lett. 83, 1566 (1999).

[6] E. Braaten and H. W. Hammer, Phys. Rev. A 70, 042706 (2004).
[7] T. Kraemer et al., Nature 440, 315 (2006).

[8] M. Zaccanti, B. Deissler, C. D’Errico, M. Fattori, M. Jona-Lasinio, S. Müller, G. Roati, M. Inguscio, and G. Modugno, Nature Phys. 5, 586 (2009).

[9] T. B. Ottenstein, T. Lompe, M. Kohnen, A. N. Wenz, and S. Jochim, Phys. Rev. Lett. 101, 203202 (2008).

[10] J. H. Huckans, J. R. Williams, E. L. Hazlett, R. W. Stites, and K. M. O'Hara, Phys. Rev. Lett. 102, 165302 (2009). 
[11] N. Gross, Z. Shotan, S. Kokkelmans, and L. Khaykovich, Phys. Rev. Lett. 103, 163202 (2009).

[12] S. E. Pollack, D. Dries, and R. G. Hulet, Science 326, 1683 (2009).

[13] J. von Stecher, J. P. D'Incao, and C. H. Greene, Nature Phys. 5, 417 (2009).

[14] F. Ferlaino, S. Knoop, M. Berninger, W. Harm, J. P. D’Incao, H. C. Nägerl, and R. Grimm, Phys. Rev. Lett. 102, 140401 (2009).

[15] U. Fano, Phys. Rev. A 24, 2402 (1981).

[16] U. Fano, Phys. Today 29, 32 (1976).

[17] C. W. Clark and C. H. Greene, Phys. Rev. A 21, 1786 (1980).

[18] J. Avery, Hyperspherical Harmonics: Applications in Quantum Theory (Kluwer Academic, Boston, MA, 1989).

[19] Y. Zhou, C. D. Lin, and J. Shertzer, J. Phys. B 26, 3937 (1993).

[20] C. D. Lin, Phys. Rep. 257, 1 (1995).

[21] V. Kokoouline and C. H. Greene, Phys. Rev. A 68, 012703 (2003).

[22] M. Fabre de la Ripelle, Few-Body Syst. 14, 1 (1993).

[23] R. Szmytkowski, J. Math. Phys. 47, 063506 (2006).

[24] Y. F. Smirnov and K. V. Shitikova, Fiz. Elem. Chastits. At. Yadra. 8, 847 (1977) [Sov. J. Part. Nucl. 8, 344 (1977)].
[25] J. D. Jackson, Classical Electrodynamics, 3rd ed. (Wiley, New York, 1999).

[26] M. Abramowitz and I. A. Stegun, Handbook of Mathematical Functions, With Formulas, Graphs, and Mathematical Tables (Dover, New York, 1965).

[27] E. Nielsen, D. V. Fedorov, A. S. Jensen, and E. Garrido, Phys. Rep. 347, 373 (2001).

[28] J. P. D'Incao and B. D. Esry, Phys. Rev. Lett. 94, 213201 (2005).

[29] E. Braaten and H.-W. Hammer, Phys. Rep. 428, 259 (2006).

[30] M. Ross and G. Shaw, Ann. Phys. (NY) 13, 147 (1961).

[31] V. Efimov, Phys. Lett. B 33, 563 (1970).

[32] O. I. Kartavtsev and A. V. Malykh, J. Phys. B 40, 1429 (2007).

[33] E. Braaten, H. W. Hammer, D. Kang, and L. Platter, Phys. Rev. Lett. 103, 073202 (2009).

[34] P. Naidon and M. Ueda, Phys. Rev. Lett. 103, 073203 (2009).

[35] J. P. D'Incao and B. D. Esry, Phys. Rev. Lett. 103, 083202 (2009).

[36] S. T. Rittenhouse, Phys. Rev. A 81, 040701(R) (2010).

[37] M. Bartenstein et al., Phys. Rev. Lett. 94, 103201 (2005).

[38] N. P. Mehta, S. T. Rittenhouse, J. P. D’Incao, J. von Stecher, and C. H. Greene, Phys. Rev. Lett. 103, 153201 (2009).

[39] R. Langer, Phys. Rev. 51, 669 (1937). 